\title{
The development of biosensor with imaging ellipsometry
}

\author{
G. Jin*, Z-Y. Zhao, Z-H. Wang, Y-H. Meng, P-Q. Ying, S. Chen, Y-Y. Chen, C. Qi, L-H. Xia \\ Institute of Mechanics, Chinese Academy of Sciences, \#15, Bei-si-huan West Rd., Beijing 100080, P.R.China \\ *Tel./Fax: +86-10-62631816, E-mail: gajin@imech.ac.cn
}

\begin{abstract}
The concept of biosensor with imaging ellipsometry was proposed about ten years ago. It has become an automatic analysis technique for protein detection with merits of label-free, multi-protein analysis, and real-time analysis for protein interaction process, etc. Its principle, and related technique units, such as micro-array, micro-fluidic and bio-molecule interaction cell, sampling unit and calibration for quantitative detection as well as its applications in biomedicine field are presented here.
\end{abstract}

Keywords - Biosensor, imaging ellipsometry, micro-array

\section{INTRODUCTION}

The biosensor concept based on imaging ellipsometry for visualization of biomolecular interactions was reported in 1995 [1, 2]. In order to develop it into a practical technique for protein analysis or protein detection, a lot of problems had to be faced, such as ligand immobilization, protein and tested sample delivery, biomolecule affinity presentation on chip, specific interaction, unspecific binding influence, detection sensitivity, sample consumption, sampling and calibration for quantitative detection, etc. It has become an automatic analysis technique for protein detection with merits of label-free, multi-protein analysis, real-time analysis for protein interaction process and quantitative analysis, etc [3].

\section{Methodology}

The biosensor principle and related techniques are presented in this section.

\section{A. Principle}

Imaging ellipsometry is used to visualize the molecule surface concentration distribution of protein layers attached on a patterned surface on a solid substrate. A ligand and its receptor such as an antibody and its corresponding antigen can assemble into complexes due to their affinity. The optical biosensor is based on that each reactant as a ligand is immobilized to a surface to form a monolayer as a bioprobe with its bioactivity. The other reactant as the analyte (or receptor) exists in a solution. The bioprobe is exposed to the solution containing analyte. When the analyte in the solution interacts with its corresponding ligand on the bioprobe and assembles into complex upon their affinity. The molecule surface concentration on the surface where the interaction takes place becomes higher than before exposure to the analyte solution. A significant increase of the layer thickness (surface concentration) indicates that the solution contained receptor against the ligand on the surface. With a visualization of imaging ellipsometry, which has a high spatial resolution in the order of 0.1 nano-meter in vertical and micron in lateral, so the increase can be determined, and in this way, the existence of the analyte in the solution can be verified. Many bioprobes arrayed in matrix are used for multi-detection [3-5].

\section{B. Surface Modification and Ligand Immobilization}

In the development of biosensors, the immobilization of biomolecules at interfaces played a crucial role. At the early stages of the biosensor, the ligand immobilization was done by physical adsorption. The use of such attachment techniques involves certain limitations. The immobilized proteins suffer partial denaturation and tend to leach or wash off the surface $[6,7]$. In some cases, the adsorbed protein is displaced from the surface by other more active protein, because of the competitive adsorption of proteins $[8,9]$. To overcome these problems, covalent immobilization is the preferred method of attaching proteins to silicon surface due to the strong, stable linkage. We investigated the feasibility of using 3-aminopropyltriethoxysilane (APTES) to modify silicon surface and then activating with glutaraldehyde to immobilize protein covalently for the biosensor based on imaging ellipsometry as an alternative to the direct physical adsorption [10]. This protein immobilization method has been used successfully in other immunoassay techniques [11-14]. In order to obtain high bioactivity of ligand on the bioprobe, the oriented immobilization of ligand has to be done. Several techniques for the surface modification and ligand immobilization have been developed.

Surface modification with dichlorodimethylsilane [10]

Surface modified with this method is highly hydrophobic, proteins immobilized mainly through hydrophobic interaction between proteins and the surfaces. The protein adsorption amount is higher than that on hydrophilic modified surfaces, but immobilized proteins will be desorbed to some extent.

Oriented immobilization of IgG by Protein A [15]

The hydrophobic surface treated with dichlorodimethylsilane is first incubated in protein A solution. The protein A modified surface is then blocked with BSA, and incubated in anti-IgG or IgG solution. Protein A can bind selectively the Fc regions of antibody. The amount of $\operatorname{IgG}$ bound with anti-IgG immobilized by the protein $A$ on silicon surface is much more than that bound with anti-IgG 
immobilized by physical adsorption. Also IgG immobilized on protein A could bind more anti-body.

\section{Modification with aminopropyltriethoxysilane (APTES)} and glutaraldehyde [10]

This method could immobilize proteins covalently. The higher density and stability of IgG layer could be obtained on the APTES and glutaraldehyde modified surface than the dichlorodimethylsilane modified surfaces.

\section{Modification with a mixed silane layer [16]}

Hydrophilic silicon surfaces were reacted with a fresh ethanol solution of aminopropyltriethoxysilane (APTES) and methyltriethoxysilane (METS) $(1: 30, \mathrm{~mol} / \mathrm{mol})$. This procedure rendered the surface medium hydrophobic. Through the reaction of glutaraldehyde with APTES, proteins could be covalently immobilized on the surfaces. Compared with the only APTES or MTES treated surfaces, the IgG covalently immobilized on the mixed silane surface could retain their structures well and bind more antibody molecules.

\section{Micro-fluidic and Bio-molecule Interaction Cell}

The importance of protein array in biological research was presented in many literatures [17-19], but most of them are analogous with DNA chips in terms of fabrication and detection. Proteins are labeled and the results are detected with fluorescence readers. Thomas Kodadek pointed out several problems of the proteins labeling [17]. First, the chemical heterogeneity of proteins makes this hopeless as a strategy for doing quantitative work. Some proteins will be labeled far more efficiently than others. Then the chemical labeling of proteins can change their surface characteristics greatly that can result in significant protein denaturation, especially for smaller proteins and peptide hormones. Finally, protein labeling is a labor-intensive processing step for a high throughput protein chip. To get quantitative and reliable result, the spots on protein chip should have a homogeneous surface and the same size. It is hardly to get perfect spots by spotting or printing technique currently used in protein array fabrication [20]. A significant timedependent slowdown of binding is often observed in protein array detection, which can be due to mass transport limitations in static conditions [21].

A kind of microfluidic system was designed for the fabrication of protein array on chemically modified silicon surface and the reaction of protein array with analyte solution [22]. By the microfluidic system, many proteins could be delivered individually to each spot of the array and immobilized covalently on surface simultaneously, but also the spots of the array could be connected in series with simple channels junction to react with one analyte solution in turn. The flow of proteins solutions in microfluidic system could minimize the mass transport limitation effectively and the time for protein array fabrication or reaction (within $30 \mathrm{~min}$.) was reduced greatly. The homogeneous spots could be obtained by this sample delivery method, which was favor of getting reliable data by the array biosensor based on imaging ellipsometry. An interesting advantage of the microfluidic system was that it could be used repeatedly for protein array fabrication and reaction, which reduced greatly the cost of protein array, test time and consumption of proteins $(15 \mu \mathrm{l})$ as well as high sensitivity (1 ng/ml for Ig) [22].

\section{Automatic System of Imaging Ellipsometry}

The array biosensor based on imaging ellipsometry was a fast and convenient detection technique for protein array. Multiple analytes could be detected simultaneously and samples could be measured directly without requiring any labeling. Imaging ellipsometry was an enhancement of standard single-beam ellipsometry, which combined the power of ellipsometry with microscopy and worked in the off-null mode. The imaging ellipsometry used in this study was an automated one, and ellipsometric conditions could be controlled by auto-adjusting the polarizer and the analyzer; the angle of incidence was variable from 45 to $90^{\circ}$ with a resolution of $0.05^{\circ}$; the magnification of image to object is modulated according to the dimension of the field of view, so that the lateral resolution of micron could be reached; autofocusing was realized with the standard of the Laplacian algorithm [23]. All the adjustments were carried out automatically with micro-stepping motors controlled by a computer with homemade software. With the same software, the digital images in gray scale format ( 8 bits, $0-255$ Gycale or 10 bits, 1024 Gycale) could be also automatically captured and processed. The light source was a xenon lamp, and a specific collimating system was used to provide an expanded parallel probe beam with a diameter of about 25 $\mathrm{mm}$. The beam passed through a polarizer and a compensator (a quarter wave plate) and finally onto the sample. An optical monochrometer with a wavelength range of $400-650 \mathrm{~nm}$ and the bandwidth of $5-10 \mathrm{~nm}$ variable was placed in the incident optical passage to select a wavelength in order to obtain a high ellipsometric contrast in images. The reflection beam passed through an analyzer and an imaging lens with a spatial filter located at its focus plane, and then the ellipsometric image was focused onto the sensing area of the CCD camera. For a sample with lateral distribution of layer thickness (or surface concentration), null ellipsometry could not be carried out over the entire surface simultaneously due to the fact that different areas would yield different polarization changes. To overcome the problem, the optical components in the biosensor were adjusted to fulfill the null conditions on a bare substrate surface without adsorbed layers, and the off-null ellipsometric principle was used to measure the biomolecule layer thickness (or surface concentration). Under this condition, the detected intensity "I" was related to the thickness (th) of the layer according to $\mathrm{I}=\mathrm{k}(\mathrm{th})^{2}[1]$. As for the same protein and the same ellipsometric conditions, $\mathrm{k}$ is 
a constant and can be determined by the protein layer with known intensity in gray scale and its absolute thickness [24].

\section{E. Calibration for Quantitative Detection}

Imaging ellipsometry may visualize protein layer with distinct graph and explain results with qualitative analysis. If one wanted to analysis protein interactions and their results quantitatively, some auxiliary experiments should be performed for calibration. Usually the refractive index of saturated protein layers in visible light region was in the range of 1.4-1.5 [25], and the related variation of layer thickness was interested, but not the absolute value of the thickness, so it's simplified that the refractive indices of protein layers were supposed same. In this case, a series of the interested protein solutions with different concentration was used to get a series of protein detection results within a certain interaction time, which resulted in a calibration curve between the ellipsometric signal and the protein concentration in the solution, so that the unknown concentration could be calibrated with the curve.

The absolute thickness of the protein layer was calibrated by conventional ellipsometry. The relationship between surface concentration and protein layer thickness was the surface concentration $\left(\mu \mathrm{g} / \mathrm{cm}^{2}\right) \approx \mathrm{Kth}(\mathrm{nm})$, where $\mathrm{K} \approx 0.12$ [26].

\section{APPLICATIONS}

Now the applications of this technique mainly focused on biomedical fields. So far the technique has been used successfully for 1) biomolecule interaction [3]; 2) hormone detection [27]; 3) cell factor and its receptor interaction [28]; 4) cancer marker test [29]; 5) mono-clone medicine evaluation [30]; 6) clinic diagnosis for hepatitis B [31]; 7) quantitative protein competitive adsorption [21,32-33]; 8) kinetic detection for multi-protein interaction process $[3,34]$, etc.

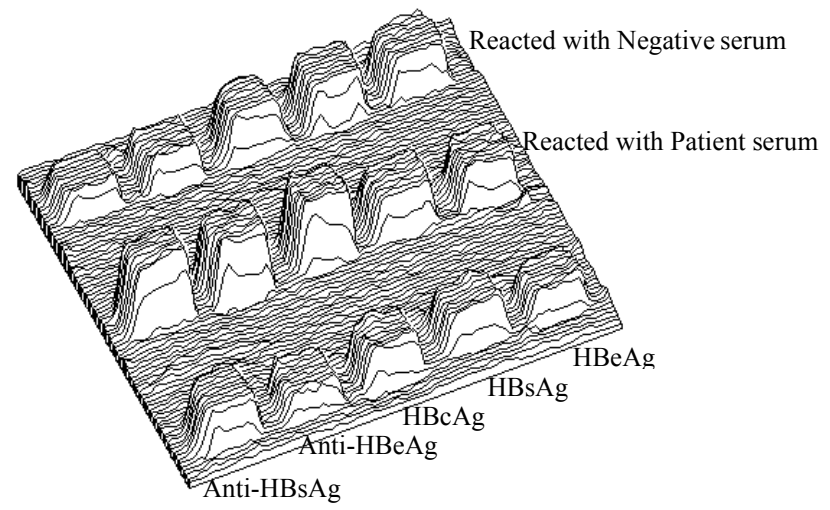

Fig. 1. The detection of five markers for Hepatitis B diagnosis, and the thickness of protein layers were directly converted by the grayscale of image.
Hepatitis $\mathrm{B}$ is a serious disease caused by a virus that attacks the liver. The virus, which is called hepatitis $B$ virus (HBV), can cause lifelong infection, the cirrhosis (scarring) of liver, the liver cancer, the liver failure, and even death. The diagnosis of HBV infection is generally made on the basis of serology. HBsAg, HBeAg, Anti-HBcAg, Anti$\mathrm{HBsAg}$ and Anti-HBeAg are five markers for Hepatitis B diagnosis. These five makers in serum can be detected on one protein chip by the technique. Fig. 1 showed a detection result of a Hepatitis B patient serum with protein chip. AntiHBsAg, Anti-HBeAg, $\mathrm{HBcAg}, \mathrm{HBsAg}$ and $\mathrm{HBeAg}$ were first immobilized covalently on protein chip in triple by microfluidic system. One spot reacted with negative serum, another spot reacted with patient serum, and the left one for reference. The result showed three makers were positive, which were $\mathrm{HBsAg}, \mathrm{HBeAg}$, and Anti-HBcAg, respectively, which was in an agreement with ELISA.

\section{CONCLUSION}

Within last ten years, the biosensor with imaging ellipsometry has developed as an automatic analysis technique for protein detection, with merits of label-free, multi-protein analysis, and real-time analysis for protein interaction process, etc. It can be foreseen that an application potential in the fields of proteomics, clinical diagnostics, environmental control and biological research.

\section{ACKNOWLEDGMENT}

The national natural science foundation of China (No. 60178033, No. 90206029) and Chinese Academy of Sciences are acknowledged for their supports.

\section{REFERENCES}

[1] G. Jin, P. Tengvall, I. Lundstršm and H. Arwin, “A Biosensor Concept Based on Imaging Ellipsometry for Visualization of Biomolecular Interactions," Analytical Biochemistry, vol.232, pp. 69-72, 1995.

[2] G. JIN, R. Jansson, and H. Arwin, “Imaging ellipsometry revisited: Developments for visualization of thin transparent layers on silicon substrates," Rev. Sci. Instrum., vol.67, pp. 2930-2936, 1996.

[3] Z. H. Wang, G. Jin, "A label-free multisensing immunosensor based on Imaging Ellipsometry," Analytical Chemistry, vol.75, no. 22, pp. 6119-6123, 2003.

[4] G. Jin, Z. H. Wang, "Micro-systems for Optical Protein-Chip," International Journal of Nonlinear Sciences and Numerical Simulation, vol.3, no. 3-4, pp. 191-194, 2002.

[5] G. Jin, Z. H. Wang, Y.H. Meng, P.Q. Ying, and L.H. Xia, "Optical ProteinChip as Microarrays for Protein Interaction Determination," In Proc. 23rd Annual International Conference of the IEEE Engineering in Medicine and Biology Society, Oct.25-28, 2001, Istanbul, Turkey. 
[6] S. K. Bhatia, L. C. Shriver-Lake, K. J. Prior, J. H. Georger, J. M. Calvert, R. Bredehorst, F. S. Ligler, "Use of thiol-terminal silanes and heterobifunctional crosslinkers for immobilization of antibodies on silica surfaces," Anal. Biochem., vol.178, no. 2, pp. 408-13, May, 1989.

[7] L. C. Shriver-Lake, B. Donner, R. Edelstein, K. Breslin, S. K. Bhatia, F. S. Ligler, "Antibody immobilization using heterobifunctional crosslinkers," Biosens. Bioelectron., vol.12, no. 11, pp. 1101-6, 1997.

[8] L. Vroman, A. L. Adams, G. C. Fischer, P. C. Munoz, "Interaction of high molecular weight kininogen, factor XII, and fibrinogen in plasma at interfaces," Blood, vol.55, no. 1, pp. 156$159,1980$.

[9] L. Vroman, A. L. Adams, "Finddings with the recording ellipsometer suggesting rapid exchange of specific plasma proteins at liquid solid interaces," Surf. Sci., vol. 16, pp. 438-446 A29, 1969.

[10] Z. Wang, G. Jin, "Covalent immobilization of proteins for the biosensor based on imaging ellipsometry," J. Immunol. Meth., vol. 285, pp. 237-243, 2004.

[11] H. Yuan, M. Wayne, Mullett, and J. Pawliszyn, "Biological sample analysis with immunoaffinity solid-phase microextraction," The Analyst, vol.126, pp. 1456-1461, 2001.

[12] J. Lin, J. Herron, J. D. Andrade, M. Brizgys, "Characterization of immobilized antibodies on silica surfaces," IEEE Transactions on biomedical engineering, vol.35, no. 6, pp. 466-71,1988.

[13] M. Yoshioka, and Y. Mukai, "Immobilization of ultra-thin layer of monoclonal antibody on glass surface," J. Chromatography, Biomedical applications, vol.566, pp. 361-368, 1991.

[14] C. M. Halliwell, and A. E. G. Cass, "A factorial analysis of silanization conditions for the immobilization of oligonucleotides on glass surfaces," Anal. Chem. Vol.73, pp. 2476-2483, 2001.

[15] Z. Wang, G. Jin, "Feasibility of protein A for the oriented immobilization of immunoglobulin on silicon surface for a biosensor with imaging ellipsometry," J. Biochem. Biophys. Methods, vol. 57, pp. 203-211, 2003.

[16] Z.H. Wang, G. Jin, "Silicon surface modification with a mixed silanes layer to immobilized proteins for biosensor with imaging ellipsometry," Colloids and Surfaces B: Biointerfaces, vol.34 no. 3, pp. 173-177, 2004.

[17] T. Kodadek, "Protein microarray: prospects and problems," Chemistry \& Biology, vol.8, pp. 105-115, 2001.

[18] A. Talapatra, R. Rouse, G. Hardiman, "Protein microarrays: challenges and promises," Pharmacogenomics, vol.3, no.4, pp. 527-536, 2002.

[19] G. MacBeath, S. L. Schreiber, "Printing proteins as microarrays for high-throughput function determination," Science, vol.289, pp. 1760-1763, 2000.

[20] M. F. Templin, D. Stoll, M. Schrenk, P. C. Traub, C. F. Vohringer, T. O. Joos, "Protein microarray technology," Drug Discov Today, vol.7, no. 15, pp. 815-22, 2002.

[21] O. Hofmann, G. Voirin, P. Niedermann, and A. Manz, "Threedimensional microfluidic confinement for efficient sample delivery to biosensor surfaces. Application to immunoassays on planar optical waveguides," Anal. Chem. Vol.74, pp. 5243-5250, 2002.

[22] Z. Wang, G. Jin, unpublished.

[23] Y. Meng, G. Jin, "The study for the autofocusing of imaging ellipsometry system," J. Test and measurement technology of NCIT, vol.14, pp. 220-225, 2000.

[24] P. Ying, Y. Yu, G. Jin and Z. Tao, "Competitive protein adsorption studied with atomic force microscopy and imaging ellipsometry," Colloids and Surfaces B: Biointerfaces, vol.32, no. 1, pp. 1-10, 2003.

[25] H. Arwin, "Optical properties of thin layers of Bovine serum albumin, $\gamma$-globulin, and hemoglobin," Applied Spectroscopy, vol.40, no.3, pp.313-318, 1986.

[26] M. Stenberg, H. Nygren, "The use of the isoscope ellipsometer in the study of adsorbed proteins and biospecificbinding reactions," J. Phys. Vol.44, pp. 83-6, 1983.
[27] Z-Y. Zhao, G. Jin, Z. H. Wang, "Detection of somatotropin and corticosterone with imaging ellipsometry," in Proc. $20^{\text {th }}$ Internation conference of the IEEE Engineering in Medicine and Bilogy Society, Hong Kong, pp. 269-272, 1998.

[28] Z. H. Wang, G. Jin, "Visualization of the interaction between IL-6 and IL-6R by imaging ellipsometry," Sheng Wu Gong Cheng Xue Bao, vol.18, no. 1, pp. 99-101, 2002.

[29] C. Qi, G. Jin, et al., unpublished.

[30] G. Jin, et al., unpublished.

[31] Z. Wang, G. Jin, unpublished.

[32] P. Ying, G. Jin and Z. Tao, "Protein Competitive Adsorption Studied with Imaging Ellipsometry," In Proc. 2nd European Medical and Biological Engineering Conference, Vienna, Austria, pp. 190-191, 2002

[33] P. Ying, G. Jin, and Z. Tao, "Competitive adsorption of collagen and bovine serum albumin - Effect of the surface wettability," Colloids and surfaces B: biointerfaces, vol.33, no. 3-4, pp. 259263, 2004.

[34] G.Jin, Z.Y.Zhao, Z.H. Wang, Y.H.Meng and L.H.Xia, "Optical Biosensor with Imaging Ellipsometry for Real-time Visualization of Bio-molecular Interaction," In Proc. IEEE conference, ISIK2000 Workshop on Biomedical Information Engineering, Istanbul, Turkey, pp. 137-140, 2000. 